\title{
INFLUÊNCIA DO SÍTIO NO DESENVOLVIMENTO DO Pinus taeda L. AOS 22 ANOS: ESTADO NUTRICIONAL DAS PLANTAS
}

\author{
Renato Antonio Dedecek ${ }^{*}$, Ivone Satsuki Namikawa Fier ${ }^{* *}$, Randy Speltz ${ }^{* * *}$, Luis Carlos de Souza Lima ${ }^{* * *}$ \\ "Eng. Agrônomo, Ph.D., Embrapa Florestas - dedecek@cnpf.embrapa.br \\ *Eng ${ }^{a}$. Florestal, M.Sc., Klabin S.A. - namikawa@klabinpr.com.br \\ ${ }_{* * *}^{*}$ Eng. Florestal, M.Sc., Klabin S.A. - randys@klabinpr.com.br \\ ${ }^{* * * *}$ Técnico Florestal, Klabin S.A. - luiscarlos@klabinpr.com.br \\ Recebido para publicação: 04/04/2007 - Aceito para publicação: 23/08/2007
}

\begin{abstract}
Resumo
A análise de solo, freqüentemente usada na agricultura, tem provado ser pouco útil em prever o crescimento de espécies florestais pela dificuldade em determinar com precisão a camada do solo de maior absorção dos nutrientes para a amostragem. Para avaliar a influência do sítio no crescimento do Pinus taeda, foram selecionados, em área de plantio comercial da Klabin S.A., no município de Telêmaco Borba (PR), oito sítios com essa espécie aos 22 anos de idade, diferenciados pelo tipo de solo (latossolo e cambissolo), textura (argilosa e média) e vegetação original (campo e floresta). Foram realizadas avaliações dendrométricas de 50 árvores por sítio, e selecionadas 3 árvores médias por sítio, nas quais foram medidas alturas totais, altura para serraria e para celulose e DAP. Também foram coletados discos em seis posições e acículas no terço superior da copa dessas árvores, para análise do estado nutricional. A concentração de $\mathrm{K}$ foi maior nas acículas, na casca e no alburno das árvores dos sítios mais produtivos. Os sítios mais produtivos apresentaram os menores teores de $\mathrm{Ca}$ nas acículas das árvores, e estes mostraram uma correlação linear positiva muito forte $(r=0,80)$ com a porosidade total do solo, evidenciando uma dependência da aeração do solo para absorção de $\mathrm{Ca}$. $\mathrm{O}$ teor de $\mathrm{Zn}$ nas acículas dos sítios mais produtivos foi muito maior e mostrou as maiores correlações lineares positivas com as variáveis de crescimento. A relação $\mathrm{P} / \mathrm{Zn}$ nas acículas das plantas apresenta correlação linear negativa forte com o crescimento das árvores.

Palavras-chave: Altura; DAP; concentração de nutrientes.
\end{abstract}

\begin{abstract}
Site influence on 22-years-old Pinus taeda L. growth: plant nutritional status. Soil analyses, frequently used on agriculture, has been proved to be useless on predicting forest species growth, considering the problem on sampling the exact soil depth from where the nutrients are obtained. In order to evaluate the influence of site quality on pine trees growth, eight different sites were selected in a commercial Pinus taeda plantation belonging to Klabin, in Telêmaco Borba region, Parana State. Trees were 22 years old and sites were selected by soil type (Latosol and Cambisol), by soil texture (clayey and sandy) and by original vegetation (forest and grass). To characterize the sites, measurements of total height and DBH were taken from at least 50 trees at each site. Three average trees were selected from each site and DBH and total, sawing and cellulose heights were measured. Disks from six different tree positions and needles were collected for nutritional status analysis. K concentration was higher in needles, bark and sapwood in trees from the most productive sites. The most productive sites presented less Ca content on needles from trees that showed strong positive linear correlation $(\mathrm{r}=0,80)$ with total soil porosity, pointing that soil aeration porosity is very important on $\mathrm{Ca}$ absorption. Trees from these sites presented higher concentration of $\mathrm{Zn}$ in the needles and showed the best negative linear correlation coefficients with the growth variables Keywords: Tree height; DBH; nutrient concentration.
\end{abstract}

\section{INTRODUÇ̃̃O}

O monitoramento da concentração dos nutrientes nas acículas e sua relação com o crescimento das árvores tem sido um importante procedimento na identificação do potencial produtivo dos diferentes sítios e na tomada de decisões sobre os ajustes da adubação. Em se tratando de diagnose nutricional das 
árvores, a análise química foliar é muito utilizada, assumindo que o teor do nutriente contido nas folhas ou acículas seja um reflexo de sua disponibilidade no solo e que aumentos ou decréscimos nas concentrações de nutrientes nas folhas se relacionam com produtividades maiores ou menores, respectivamente (REISSMANN, 2002; BELLOTE; SILVA, 2000).

Segundo Schoenholtz et al. (2000), muitos métodos de análise de solo freqüentemente usados na agricultura têm provado ser pouco úteis em prever o crescimento de espécies florestais. A maior dificuldade está em determinar com precisão a camada do solo de maior absorção dos nutrientes e da água, para se efetuar a amostragem (MARSCHNER, 1995). Para Silveira et al. (2000), a análise foliar é o critério mais apropriado para determinar o estado nutricional das plantas perenes, devendo os nutrientes estar contidos nas folhas, não somente em concentração adequada, mas também numa relação adequada entre eles. Gonçalves (1995) observou que, embora as análises de solo tenham se constituído na forma mais prática e viável de avaliar a fertilidade do solo, há diversas dificuldades quanto à interpretação dos seus resultados. Essa limitação está relacionada principalmente com o fluxo de nutrientes que ocorre via ciclagem de nutrientes, o qual pode atender grande parte da demanda de nutrientes das árvores.

É importante considerar o crescimento da planta e a disponibilidade de nutrientes no solo na interpretação dos resultados da análise nutricional. Isso porque o teor de nutrientes no tecido vegetal é resultante da combinação de vários processos, como o suprimento externo de nutrientes, a absorção pela planta, a redistribuição e o crescimento. Sendo assim, se o crescimento aumenta mais que a absorção, então o resultado apresentará teores mais baixos, fenômeno chamado de efeito de diluição (TAMM, 1964).

Para o gênero Pinus, cada um dos compartimentos que formam a árvore possui uma concentração de nutrientes minerais que está relacionada com suas funções. Geralmente, a seqüência de concentração tem a seguinte ordem decrescente: acícula $>$ casca $>$ ramo $>$ tronco (BELLOTE; SILVA, 2000). Segundo Gonçalves (1995), a ordem dos nutrientes mais acumulados em Pinus é $\mathrm{N}>\mathrm{K}>\mathrm{Ca}>\mathrm{Mg}>\mathrm{P}$.

Para Vogel (2003), os nutrientes mais limitantes ao crescimento das plantas foram o nitrogênio e o cálcio, indicando a seguinte ordem decrescente de limitação: $\mathrm{Ne} \mathrm{Ca}>\mathrm{Mg}>\mathrm{K}>\mathrm{P}$. Os teores ótimos estimados de nitrogênio, cálcio, magnésio, potássio e fósforo nas acículas foram de 14,6;2,6;0,8; 8,7;1,6 g. $\mathrm{kg}^{-1}$, respectivamente. Menegol (1991), em estudos feitos na região dos Campos Gerais do Paraná, constatou que os baixos teores foliares de magnésio e zinco foram associados ao baixo crescimento em altura de Pinus elliottii, sendo considerados como limitantes ao crescimento dessa espécie. Já os teores foliares de nitrogênio, magnésio, cobre, potássio e zinco foram os mais correlacionados com a altura dominante aos 15 anos de idade. Para Reissmann (2002), estudos feitos no segundo planalto paranaense têm mostrado que o macronutriente potássio e o micronutriente zinco parecem ser os mais limitantes para o crescimento de Pinus taeda. Teores foliares de 1,8 $\mathrm{g} / \mathrm{kg}$ de fósforo, $6 \mathrm{~g} / \mathrm{kg}$ de potássio e $0,6 \mathrm{~g} / \mathrm{kg}$ de magnésio se correlacionaram com as maiores alturas dominantes em árvores com 15 anos de idade nessa mesma região.

Zöttl; Tschinkel (1971) citam teores de nutrientes nas acículas de espécies de Pinus considerados como adequados: 15 a 25 g. $\mathrm{kg}^{-1}$ de nitrogênio, 1,3 a 2,0 g. $\mathrm{kg}^{-1}$ de fósforo, 6 a $12 \mathrm{~g}$. $\mathrm{kg}^{-1}$ de potássio, 1 a 5 g. $\mathrm{kg}^{-1}$ de cálcio e 1 a 2 g. $\mathrm{kg}^{-1}$ de magnésio. Os teores considerados deficientes para essa espécie são inferiores a $12 \mathrm{~g} \cdot \mathrm{kg}^{-1}$ de nitrogênio, $0,9 \mathrm{~g} \cdot \mathrm{kg}^{-1}$ de fósforo, $4 \mathrm{~g} \cdot \mathrm{kg}^{-1}$ de potássio, $0,5 \mathrm{~g} \cdot \mathrm{kg}^{-1}$ de cálcio e 0,5 g.kg ${ }^{-1}$ de magnésio. Reissmann (2002), estudando Pinus taeda com mais de 20 anos de idade, também apontou como importantes para o crescimento das árvores as relações N/K e P/Zn.

Este trabalho objetiva identificar quais os elementos limitantes ou potenciais para a elaboração de programas de manejo de plantações de Pinus taeda visando a produção de madeira, ou mesmo para a elevação de sítios de classe 3 para 2 ou até para a classe mais produtiva.

\section{MATERIAL E MÉTODOS}

As amostras foram obtidas em plantios comerciais de Pinus taeda estabelecidos em 1978, localizados em áreas da Klabin S.A., em Telêmaco Borba (PR), a 240ㅇ' latitude Sul e 50³0' longitude Oeste, com altitude de 750 a $868 \mathrm{~m}$.

$\mathrm{O}$ clima da região, segundo a classificação de Köppen, é $\mathrm{Cfa} / \mathrm{Cfb}$, subtropical úmido transicional para temperado propriamente dito, em que a temperatura média do mês mais frio é inferior a $16{ }^{\circ} \mathrm{C}$, com ocorrência de geada, e a temperatura média do mês mais quente é superior a $22^{\circ} \mathrm{C}$. A precipitação média anual é de $1490 \mathrm{~mm}$, com excedente hídrico de $557 \mathrm{~mm} / \mathrm{ano}$, distribuído em todos os meses do ano (EMBRAPA/IAPAR, 1984).

$\mathrm{O}$ material de origem da região é predominantemente resultante do intemperismo e retrabalhamento de litologias referentes às formações Rio Bonito, Itararé e Diques de Diabásio (EMBRAPA/IAPAR, 1984). 
A cobertura vegetal primitiva era representada pelo contato estepe/floresta, sendo 51\% formações florestais e 49\% formações campos (EMBRAPA/IAPAR, 1984).

Os sítios foram selecionados pela vegetação original (campo e floresta), pelo tipo de solo (Latossolo e Cambissolo) e pela textura (argiloso/muito argiloso e média).

O espaçamento inicial de plantio foi de 2,5 por $2,5 \mathrm{~m}$, sendo realizado no oitavo ano um desbaste sistemático de $20 \%$, e dois desbastes seletivos no décimo segundo e décimo sexto anos, também de $20 \%$ cada um. Para a caracterização dos sítios, foram realizadas avaliações dendrométricas de 50 árvores por sítio e selecionaram-se três árvores médias (altura e DAP) por sítio, nas quais foram coletados discos em seis posições do tronco (base, DAP, $25 \%, 50 \%, 75 \%$ e $100 \%$ da altura comercial) e acículas no terço médio superior e no primeiro lançamento das árvores para análise do estado nutricional. Foi analisado o estado nutricional das plantas, por compartimento - folha, fuste e casca (SARRUGE; HAAG, 1974) -, sendo que os dados dos nutrientes no fuste e na casca referem-se à média dos seis discos obtidos por árvore.

Foram coletadas amostras de solo indeformadas e a granel em três profundidades: 0 a 10,10 a 20 e 20 a $30 \mathrm{~cm}$, a $50 \mathrm{~cm}$ de cada árvore amostrada. Nessas amostras de solo, foram feitas análises químicas (macroelementos, CTC e matéria orgânica) e físico-hídricas (densidade, porosidades, curva de retenção de água, disponibilidade de água e profundidade efetiva), seguindo metodologia descrita em Embrapa (1997). Nas tabelas 1 e 2 são apresentados dados das características físico-hídricas e químicas da camada de 0 a $10 \mathrm{~cm}$ dos solos dos sítios estudados.

Tabela 1. Características físico-hídricas do solo dos oito sítios classificados pela altura de madeira para serraria, na camada de 0 a $10 \mathrm{~cm}$.

Table 1. Soil physical characteristics for eight sites classified by saw wood height, in the 0 to $10 \mathrm{~cm}$ depth.

\begin{tabular}{|c|c|c|c|c|c|c|c|}
\hline \multirow{3}{*}{ Solo } & \multirow{3}{*}{ Textura } & \multirow{3}{*}{$\begin{array}{c}\text { Vegetação } \\
\text { original }\end{array}$} & \multirow{3}{*}{$\begin{array}{c}\text { Densidade } \\
\text { global } \\
\mathbf{k g ~ d m}^{-3}\end{array}$} & \multicolumn{2}{|c|}{ Porosidade } & \multirow{2}{*}{$\begin{array}{c}\text { Umidade } \\
\text { atual }\end{array}$} & \multirow{2}{*}{$\begin{array}{r}\text { Água } \\
\text { dispon. }\end{array}$} \\
\hline & & & & Total & Macro & & \\
\hline & & & & \multicolumn{4}{|c|}{$\mathrm{cm}^{3} \mathrm{~cm}^{-3}$} \\
\hline Cambis & média & floresta & 1,308 & 0,508 & 0,117 & 0,288 & 0,040 \\
\hline Latos & argilosa & floresta & 1,087 & 0,601 & 0,196 & 0,333 & 0,024 \\
\hline Cambis & argilosa & floresta & 1,293 & 0,518 & 0,133 & 0,341 & 0,032 \\
\hline Latos & argilosa & campo & 1,200 & 0,631 & 0,163 & 0,328 & 0,019 \\
\hline Cambis & argilosa & campo & 1,136 & 0,594 & 0,153 & 0,332 & 0,026 \\
\hline Latos & média & floresta & 0,914 & 0,687 & 0,168 & 0,352 & 0,026 \\
\hline Latos & média & campo & 0,896 & 0,674 & 0,386 & 0,149 & 0,027 \\
\hline Cambis & média & campo & 1,162 & 0,582 & 0,266 & 0,117 & 0,021 \\
\hline
\end{tabular}

Fonte: Dedecek et al., 2006. Cambis= Cambissolo; Latos= Latossolo.

Tabela 2. Características químicas dos solos dos oito sítios classificados pela altura de madeira para serraria, na camada de 0 a $10 \mathrm{~cm}$ de profundidade.

Table 2. Soil chemical characteristics for eight sites classified by saw wood height, in the 0 to $10 \mathrm{~cm}$ depth.

\begin{tabular}{|c|c|c|c|c|c|c|c|}
\hline \multirow[t]{2}{*}{ Solo } & \multirow[t]{2}{*}{ Textura } & \multirow{2}{*}{$\begin{array}{c}\text { Vegetação } \\
\text { original }\end{array}$} & $\begin{array}{c}\mathrm{pH} \\
\mathrm{CaCl}_{2}\end{array}$ & $\mathbf{K}$ & $\mathrm{Ca}+\mathrm{Mg}$ & \multirow{2}{*}{$\begin{array}{c}P \\
\text { mg.dm }\end{array}$} & \multirow{2}{*}{$\begin{array}{c}\text { Matéria } \\
\text { orgânica } \\
\text { g.kg-1 }\end{array}$} \\
\hline & & & \multicolumn{3}{|c|}{$\mathrm{cmol}_{\mathrm{c}} \cdot \mathrm{dm}^{-3}$} & & \\
\hline Cambis & média & floresta & 3,45 & 0,06 & 0,47 & 5,6 & 9,3 \\
\hline Latos & argilosa & floresta & 3,41 & 0,05 & 0,66 & 4,9 & 8,3 \\
\hline Cambis & argilosa & floresta & 3,80 & 0,20 & 0,64 & 8,9 & 19,2 \\
\hline Latos & argilosa & campo & 3,44 & 0,06 & 0,46 & 4,8 & 10,0 \\
\hline Cambis & argilosa & campo & 3,22 & 0,03 & 0,40 & 4,6 & 21,1 \\
\hline Latos & média & floresta & 3,51 & 0,03 & 0,50 & 4,9 & 18,9 \\
\hline Latos & média & campo & 3,50 & 0,15 & 0,48 & 5,6 & 5,8 \\
\hline Cambis & média & campo & 3,31 & 0,07 & 0,40 & 7,3 & 18,3 \\
\hline
\end{tabular}

Fonte: Dedecek et al., 2006. Cambis= Cambissolo; Latos= Latossolo.

Os resultados das variáveis estudadas foram submetidos à análise de variância e ao teste de Tukey. Foi utilizado o modelo de regressão linear simples e calculados os coeficientes de correlação para se entender as relações existentes entre as variáveis estudadas. 


\section{RESULTADOS E DISCUSSÃO}

Houve diferença significativa de concentração de macronutrientes nos três compartimentos avaliados (acículas, casca e alburno) de Pinus taeda apenas para K (Tabela 3). A concentração de Ca foi diferente na casca e no alburno, e o $\mathrm{N}$ apenas no alburno. A concentração de $\mathrm{K}$ nas acículas das árvores crescendo em Cambissolo textura argilosa fase floresta não foi a maior, mas também não diferiu da maior concentração apresentada. Já na casca, árvores desse solo apresentaram maior concentração de K, e no alburno esteve entre as maiores. Ainda em relação ao K, o solo, que proporcionou o maior DAP e conseqüentemente maior volume cúbico (Cambissolo textura média fase floresta), apresentou concentração entre as maiores observadas (Tabela 3), em todos os compartimentos analisados. Reissmann; Wisnewski (2000) enfatizam que a deficiência de K está associada à falta de água no solo, pela maior mobilidade dos íons de $\mathrm{K}$ em solo com maiores teores de água. Conforme Dedecek et al. (2007), a variável físico-hídrica que apresentou a melhor correlação com o crescimento do $P$. taeda foi o teor de água disponível no solo. Foi observado que o sítio com as maiores médias de crescimento que apresentaram os maiores teores de $\mathrm{K}$ nos compartimentos da planta, também foi o que apresentou os maiores valores de água disponível no solo (Tabela 1). Para Hoyle; Mader (1964), o crescimento da área basal de Pinus resinosa, nos Estados Unidos, correlacionou-se com os níveis de $\mathrm{K}$ nas acículas e o crescimento em volume correlacionou-se principalmente ao armazenamento de água no solo.

Tabela 3. Concentração de macronutrientes, em g.kg ${ }^{-1}$, nas acículas, casca e alburno de Pinus taeda com 22 anos de idade, classificados pela altura de madeira para serraria.

Table 3. Macronutrient concentration $\left(\mathrm{g}_{\mathrm{kg}} \mathrm{kg}^{-1}\right)$ in 22 years old Pinus taeda needles, bark and sapwood, ranking by saw wood height.

\begin{tabular}{|c|c|c|c|c|c|c|c|}
\hline Solo & Textura & $\begin{array}{c}\text { Vegetação } \\
\text { original }\end{array}$ & $\mathbf{N}$ & $\mathbf{P}$ & $\mathbf{K}$ & $\mathrm{Ca}$ & Mg \\
\hline \multicolumn{8}{|c|}{ Acículas } \\
\hline Cambissolo & média & floresta & $16,04 \mathrm{a}$ & $1,10 \mathrm{a}$ & $4,74 \mathrm{ab}$ & $1,31 \mathrm{a}$ & $0,80 \mathrm{a}$ \\
\hline Latossolo & argilosa & floresta & $16,61 \mathrm{a}$ & $1,01 \mathrm{a}$ & $3,74 \mathrm{bc}$ & $0,98 \mathrm{a}$ & $0,80 \mathrm{a}$ \\
\hline Cambissolo & argilosa & floresta & $16,62 \mathrm{a}$ & $0,95 \mathrm{a}$ & $4,31 \mathrm{abc}$ & $0,83 \mathrm{a}$ & $0,55 \mathrm{a}$ \\
\hline Latossolo & argilosa & campo & $18,16 \mathrm{a}$ & $1,05 \mathrm{a}$ & $3,00 \mathrm{c}$ & $2,55 \mathrm{a}$ & $0,86 \mathrm{a}$ \\
\hline Cambissolo & argilosa & campo & $18,07 \mathrm{a}$ & $1,03 \mathrm{a}$ & $5,47 \mathrm{a}$ & $1,81 \mathrm{a}$ & $0,51 \mathrm{a}$ \\
\hline Latossolo & média & floresta & $18,76 \mathrm{a}$ & $1,08 \mathrm{a}$ & $3,96 \mathrm{abc}$ & $2,23 \mathrm{a}$ & $0,60 \mathrm{a}$ \\
\hline Latossolo & média & campo & $17,53 \mathrm{a}$ & $1,11 \mathrm{a}$ & $3,51 \mathrm{bc}$ & $2,72 \mathrm{a}$ & $0,75 \mathrm{a}$ \\
\hline Cambissolo & média & campo & $17,72 \mathrm{a}$ & $1,12 \mathrm{a}$ & $4,16 \mathrm{abc}$ & $1,73 \mathrm{a}$ & $0,76 \mathrm{a}$ \\
\hline C.V. $(\%)$ & & & 10,2 & 8,4 & 13,5 & 59,4 & 18,2 \\
\hline \multicolumn{8}{|c|}{ Casca } \\
\hline Cambissolo & média & floresta & $4,14 \mathrm{a}$ & $0,29 \mathrm{a}$ & $1,09 \mathrm{a}$ & $0,77 \mathrm{~b}$ & $0,53 \mathrm{a}$ \\
\hline Latossolo & argilosa & floresta & $3,64 \mathrm{a}$ & $0,17 \mathrm{a}$ & $0,66 \mathrm{abc}$ & $0,25 \mathrm{~b}$ & $0,20 \mathrm{a}$ \\
\hline Cambissolo & argilosa & floresta & $3,84 \mathrm{a}$ & $0,17 \mathrm{a}$ & $0,89 \mathrm{ab}$ & $0,80 \mathrm{~b}$ & $0,55 \mathrm{a}$ \\
\hline Latossolo & argilosa & campo & $2,96 \mathrm{a}$ & $0,17 \mathrm{a}$ & $0,31 \mathrm{c}$ & $0,92 \mathrm{ab}$ & $0,43 \mathrm{a}$ \\
\hline Cambissolo & argilosa & campo & $2,84 \mathrm{a}$ & $0,17 \mathrm{a}$ & $0,66 \mathrm{abc}$ & $0,98 \mathrm{a}$ & $0,34 \mathrm{a}$ \\
\hline Latossolo & média & floresta & $3,10 \mathrm{a}$ & $0,18 \mathrm{a}$ & $0,65 \mathrm{abc}$ & $1,56 \mathrm{a}$ & $0,31 \mathrm{a}$ \\
\hline Latossolo & média & campo & $3,64 \mathrm{a}$ & $0,26 \mathrm{a}$ & $0,42 \mathrm{bc}$ & $1,02 \mathrm{a}$ & $0,51 \mathrm{a}$ \\
\hline Cambissolo & média & campo & $3,32 \mathrm{a}$ & $0,16 \mathrm{a}$ & $0,43 \mathrm{bc}$ & $0,89 \mathrm{ab}$ & $0,30 \mathrm{a}$ \\
\hline C.V. $(\%)$ & & & 12,3 & 27,1 & 27,6 & 26,3 & 31,3 \\
\hline \multicolumn{8}{|c|}{ Alburno } \\
\hline Cambissolo & média & floresta & $0,79 \mathrm{~b}$ & $0,06 \mathrm{a}$ & $0,45 \mathrm{a}$ & $0,63 \mathrm{ab}$ & $0,23 \mathrm{a}$ \\
\hline Latossolo & argilosa & floresta & $0,84 \mathrm{~b}$ & $0,03 \mathrm{a}$ & $0,33 \mathrm{~b}$ & $0,49 \mathrm{ab}$ & $0,19 \mathrm{a}$ \\
\hline Cambissolo & argilosa & floresta & $0,98 \mathrm{a}$ & $0,04 \mathrm{a}$ & $0,43 \mathrm{a}$ & $0,63 \mathrm{ab}$ & $0,19 \mathrm{a}$ \\
\hline Latossolo & argilosa & campo & $0,72 \mathrm{~b}$ & $0,05 \mathrm{a}$ & $0,31 \mathrm{~b}$ & $0,50 \mathrm{ab}$ & $0,14 \mathrm{a}$ \\
\hline Cambissolo & argilosa & campo & $1,12 \mathrm{a}$ & $0,06 \mathrm{a}$ & $0,47 \mathrm{a}$ & $0,39 \mathrm{~b}$ & $0,16 \mathrm{a}$ \\
\hline Latossolo & média & floresta & $1,02 \mathrm{a}$ & $0,04 \mathrm{a}$ & $0,34 \mathrm{ab}$ & $0,40 \mathrm{~b}$ & $0,13 \mathrm{a}$ \\
\hline Latossolo & média & campo & $0,87 \mathrm{ab}$ & $0,03 \mathrm{a}$ & $0,34 \mathrm{ab}$ & $0,55 \mathrm{ab}$ & $0,18 \mathrm{a}$ \\
\hline Cambissolo & média & campo & $0,93 \mathrm{ab}$ & $0,05 \mathrm{a}$ & $0,28 \mathrm{~b}$ & $0,67 \mathrm{a}$ & $0,31 \mathrm{a}$ \\
\hline C.V. (\%) & & & 9,8 & 19,9 & 12,7 & 15,9 & 30,2 \\
\hline
\end{tabular}


Considerando-se a concentração de Ca na casca, onde normalmente ocorrem em torno de 50\% do total de Ca das árvores, os dois solos que proporcionaram maior altura para serraria e DAP das árvores (Cambissolo textura argilosa fase floresta e Cambissolo textura média fase floresta, respectivamente) apresentaram os menores valores. Hoyle; Mader (1964) observaram forte correlação entre o crescimento em altura com níveis de Ca nas acículas de P. resinosa. A menor absorção de Ca nessas árvores pode ter contribuído para uma maior absorção de $\mathrm{K}$, permitindo um maior crescimento nesses sítios. Tem se observado em experimentos de casa de vegetação com diferentes níveis de água, que a absorção de Ca é maior em solo com teor de umidade adequado e principalmente com porosidade de aeração elevada (SANTANA et al., 2003). Dedecek et al. (2000) relataram também uma correlação negativa do teor de Ca nas acículas de Pinus taeda e valores de resistência do solo ao penetrômetro. Foi observado que esses dois solos, que apresentam menores teores de Ca na casca e nas acículas, exceto no alburno, também apresentaram as menores macroporosidades (Tabela 1), abaixo do limite mínimo para permitir o crescimento das raízes, entre 10 e 15\% (WEAVER; JAMISON, 1951). As correlações observadas entre os teores de $\mathrm{Ca}$ nas acículas e os valores de porosidade total e macroporosidade foram positivas e situaram-se acima de $\mathrm{r}=0,8$, nas três profundidades de solo amostradas (DEDECEK et al. 2007).

$\mathrm{Na}$ figura 1, estão os coeficientes de correlação obtidos das regressões lineares entre os teores de Ca nas acículas das árvores dos oito sítios estudados e os valores de porosidade total dos solos desses sítios nas três profundidades avaliadas (DEDECEK et al. 2007). Figura muito semelhante pode ser obtida usando-se os dados de macroporosidade do solo, e com correlações lineares negativas se os teores de $\mathrm{Ca}$ nas acículas são correlacionados com a microporosidade do solo. Acrescente-se que os teores de $\mathrm{Ca}+\mathrm{Mg}$ de todos os solos foram considerados muito baixos (Tabela 1). Apenas entre os sítios mais produtivos esses teores estão entre os mais altos. Segundo Fiedler et al. (1973), em solos com maiores teores de água há maior facilidade de absorção do K, que é também facilitada quanto maior for o teor desse nutriente em relação a $\mathrm{Ca}$ e $\mathrm{Mg}$. Observa-se na tabela 3 que mesmo no sítio menos produtivo esta relação $\mathrm{K} / \mathrm{Ca}$ é maior do que 1, quociente tido como limitante, e acima de 3 nos sítios mais produtivos.

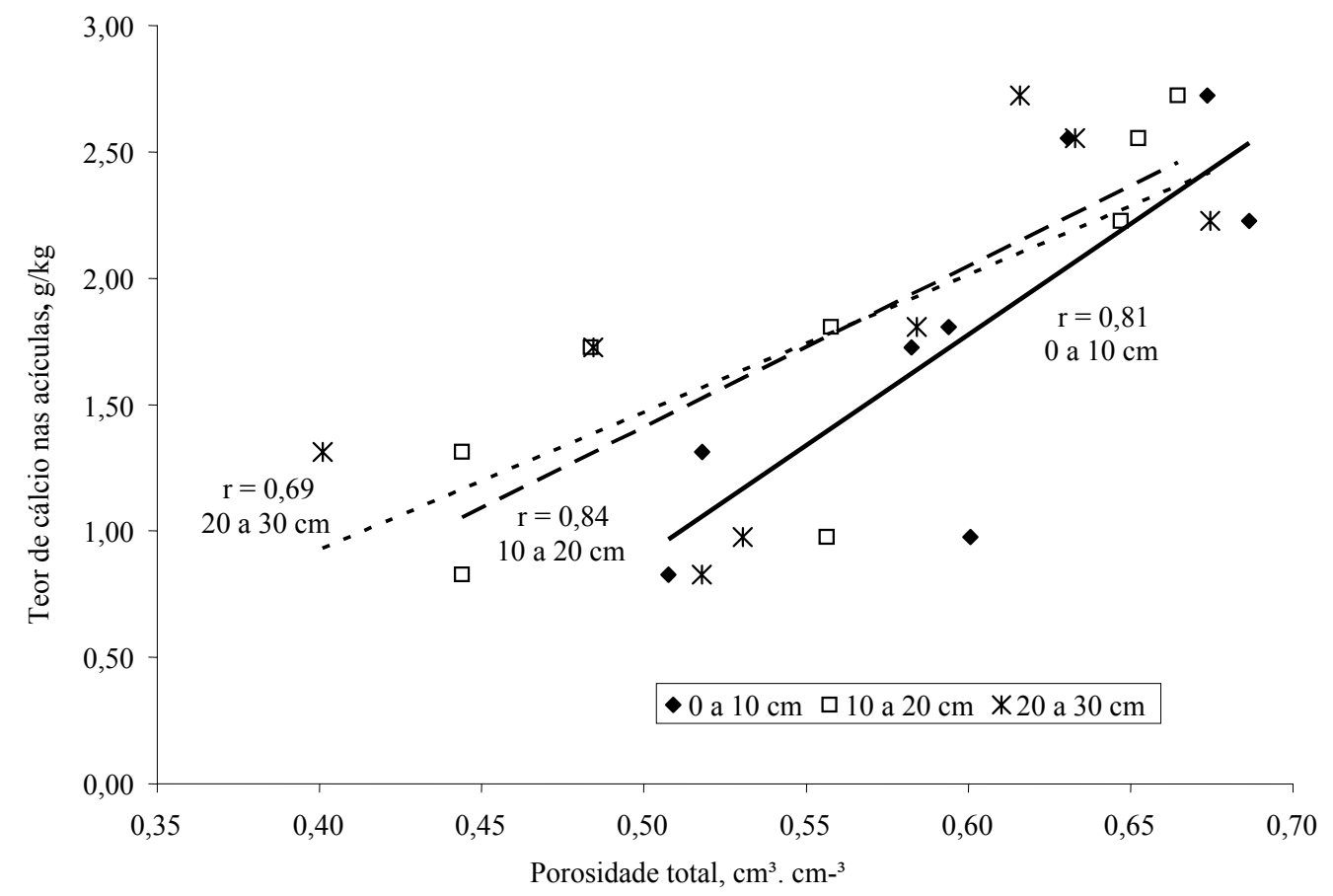

Figura 1. Regressão linear entre o teor de cálcio nas acículas de Pinus taeda e a porosidade total do solo em três profundidades para os oito sítios estudados.

Figure 1. Linear regression between Ca content in Pinus taeda needles and soil total porosity at three depths for eight different sites. 
Considerando os teores de nutrientes nas acículas de Pinus taeda com oito anos de idade apresentados por Zöttl; Tschinkel (1971), apenas os teores de Ca podem ser considerados como bons, estando todos os demais nutrientes em concentrações tidas como deficientes, embora, no solo, os teores de $\mathrm{Ca}+\mathrm{Mg}$ tenham sido considerados muito baixos (Tabela 2). Segundo Reissmann; Wisnewski (2000), as necessidades de $\mathrm{K}$ são muito superiores àquelas do Ca nessa espécie. É interessante observar ainda que o teor de P, que no solo foi considerado alto (Tabela 2), apareceu em quantidade deficiente para essa espécie nas acículas.

Para solos com teores médios de areia entre 30 e 70\%, Reissmann; Wisnewski (2000) relacionam como teores médios nas acículas das árvores com 15 anos de idade com melhores crescimentos em altura: 1,5 g. $\mathrm{kg}^{-1}$ de P, 6 g. $\mathrm{kg}^{-1}$ de K, 1,3 g. $\mathrm{kg}^{-1}$ de Ca e 0,7 g. $\mathrm{kg}^{-1}$ de $\mathrm{Mg}$. Também apenas o Ca em acículas da maioria dos sítios mostrou valores semelhantes, estando todos os demais nutrientes abaixo desses valores.

A concentração de $\mathrm{Mn}$ nas acículas, na casca e no alburno apresentou diferenças significativas para árvores procedentes dos diferentes sítios estudados, apesar de coeficientes de variação elevados (Tabela 4). Os solos mais produtivos foram também os que apresentaram os maiores teores de Mn nas acículas; já nos outros compartimentos das plantas não foi observada uma correlação entre concentração de $\mathrm{Mn}$ e crescimento. A concentração de Fe também apresentou diferenças apenas nas acículas, e não se evidenciou nenhuma correlação com as variáveis de crescimento das plantas. Em geral, altas concentrações de $\mathrm{Fe}$ e $\mathrm{Mn}$ estão freqüentemente associadas a valores baixos de pH (KABATAPENDIAS; PENDIAS, 1992). Conforme os mesmos autores, deve ser enfatizado que o conteúdo de Mn nas plantas não é apenas um efeito de suas características, mas também do 'pool' disponível de Mn, que é altamente controlado através das propriedades do solo.

Segundo Malavolta, (1980), o Mn é o segundo elemento mais abundante no solo, sendo inferior apenas ao Fe. Porém, se porventura ocorrer a elevação de uma unidade do pH do solo, conseqüentemente diminui-se em 100 vezes a concentração desse elemento na solução do solo. $O$ ferro se encontra disponível na solução do solo principalmente quelatizado, sendo que a maior parte se encontra insolúvel, não absorvível pelas raízes. Outro aspecto importante que deve ser considerado em relação à absorção do Fe é a influência que outros cátions exercem sobre ele, entre os quais o $\mathrm{Ca}, \mathrm{o} \mathrm{Mg}$, o $\mathrm{Cu}, \mathrm{o} \mathrm{Zn} \mathrm{e}$ principalmente o Mn. Devido à sua alta concentração em solos ácidos, o Mn pode inibir competitivamente a absorção do ferro causando sintomas de deficiências nas plantas (MALAVOLTA, 1980). Segundo Chaves; Correa (2003) os sintomas visuais de amarelecimento das acículas e a morte precoce de pinheiros revelam haver deficiência de manganês no sistema solo-planta estudado.

A concentração de $\mathrm{Zn}$ apresentou diferenças significativas na casca do Pinus taeda, sendo que o Cambissolo textura argilosa fase floresta que proporcionou o maior crescimento em altura é o solo com as melhores características químicas (inclusive maior teor de $\mathrm{P}$ na camada de 0 a $10 \mathrm{~cm}$ ) (Tabela 2), apresentando também árvores com os teores mais elevados de $\mathrm{Zn}$ nesse compartimento. O sítio menos produtivo, Cambissolo textura média fase campo, apresentou os menores teores de $\mathrm{Zn}$ na casca, menos que um terço do Zn presente na casca do sítio com maior crescimento em altura. Segundo Reissmann; Wisnewski (2000), Zn mostrou alta correlação com o crescimento de Pinus taeda, na região do Segundo Planalto Paranaense, onde esse estudo também foi conduzido, e pode ser considerado como um dos mais limitantes micronutrientes. Os mesmos autores citam uma correlação negativa muito forte $\mathrm{P} / \mathrm{Zn}$ nas acículas com o crescimento de árvores dessa espécie. Acrescentam ainda que, apesar do teor de P ser muito baixo nesses solos, essa correlação foi observada e que é mais comum em solos sob condições de altos níveis de P. Considerando as variáveis de crescimento (DEDECEK et al., 2007), as correlações obtidas com a relação P/Zn neste estudo também foram muito fortes e todas negativas, variando o coeficiente de correlação (r) de um mínimo de $-0,83$ com a altura total a um máximo de -0,95 com altura para serraria (Tabela 5). Ainda segundo Reissmann; Wisnewski (2000), para povoamentos de Pinus taeda com mais de 15 anos o limite inferior do bom nível de concentração de $\mathrm{Zn}$ nas acículas situa-se a partir de $30 \mathrm{mg} / \mathrm{kg}$. Observando-se a tabela 3, apenas os sítios que apresentaram maior volume de madeira e maior altura das árvores ultrapassaram esse limite de concentração de Zn nas acículas.

Poucas correlações entre as variáveis de crescimento e o estado nutricional dos compartimentos das plantas de Pinus taeda foram expressivas a ponto de contribuir em mais de $50 \%$ para a variação observada no desenvolvimento das plantas nos diferentes sítios estudados. Rigatto et al. (2005) também não observaram nenhuma correlação entre a produtividade e o estado nutricional das acículas de Pinus taeda com oito anos, trabalhando na mesma região desse estudo. As maiores correlações foram encontradas com o estado nutricional das acículas, principalmente para o teor de $\mathrm{P}$ e de $\mathrm{Zn}$ e da relação P/Zn (Tabela 5). Pode ter ocorrido uma 'deficiência induzida pelo fósforo', pois se sabe que altos níveis de fósforo no meio (solo ou solução nutritiva) causam diminuição na absorção do zinco (MALAVOLTA, 1980). Como pode ser observado na tabela 5, todas as alturas e o DAP se correlacionaram positiva e fortemente com o teor de $\mathrm{Zn}$ nas acículas, e negativamente com a relação $\mathrm{P} / \mathrm{Zn}$, embora a concentração de Zn não tenha apresentado diferenças significativas nas acículas dos diferentes sítios (Tabela 4). Reissmann; Wisnewski (2000) citam que o P inativaria determinadas enzimas dependentes do Zn. Nos 
casos de adubação com $\mathrm{P}$, deve-se considerar a aplicação conjunta de $\mathrm{Zn}$ como estratégica, dada a forte correlação negativa que a relação $\mathrm{P} / \mathrm{Zn}$ apresentou com as variáveis de crescimento. O teor de $\mathrm{Zn}$ nas acículas apresentou ainda altas correlações negativas com os valores de porosidade total e macroporosidade, e positiva com a densidade do solo, exatamente o oposto das correlações da concentração de Ca com essas variáveis físico-hídricas.

Tabela 4. Concentração de micronutrientes, em mg.kg ${ }^{-1}$, nas acículas, na casca e no alburno de Pinus taeda com 22 anos de idade.

Table 4. Micronutrient concentration, $\mathrm{mg} \cdot \mathrm{kg}^{-1}$, in 22 years old Pinus taeda needles, bark and sapwood.

\begin{tabular}{|c|c|c|c|c|c|c|}
\hline Solo & Textura & $\begin{array}{l}\text { Vegetação } \\
\text { original }\end{array}$ & $\mathbf{C u}$ & $\mathbf{F e}$ & Mn & Zn \\
\hline \multicolumn{7}{|c|}{ Acícula } \\
\hline Cambissolo & média & floresta & 4 & $85 \mathrm{c}$ & $123 \mathrm{ab}$ & $31 \mathrm{a}$ \\
\hline Latossolo & argilosa & floresta & 4 & $90 \mathrm{c}$ & $136 \mathrm{a}$ & $26 \mathrm{a}$ \\
\hline Cambissolo & argilosa & floresta & 3 & 94 bc & $157 \mathrm{a}$ & $32 \mathrm{a}$ \\
\hline Latossolo & argilosa & campo & 4 & $150 \mathrm{ab}$ & $166 \mathrm{a}$ & $26 \mathrm{a}$ \\
\hline Cambissolo & argilosa & campo & 4 & 99 abc & $47 \mathrm{bc}$ & $22 \mathrm{a}$ \\
\hline Latossolo & média & floresta & 5 & $156 \mathrm{a}$ & $34 \mathrm{c}$ & $19 \mathrm{a}$ \\
\hline Latossolo & média & campo & 4 & 96 bc & 89 abc & $18 \mathrm{a}$ \\
\hline Cambissolo & média & campo & 4 & $89 \mathrm{c}$ & $106 \mathrm{abc}$ & $20 \mathrm{a}$ \\
\hline C.V. $(\%)$ & & & - & 19,1 & 28,7 & 33,0 \\
\hline \multicolumn{7}{|c|}{ Casca } \\
\hline Cambissolo & média & floresta & $3 a$ & $53 \mathrm{a}$ & $87 \mathrm{abc}$ & $21 \mathrm{~b}$ \\
\hline Latossolo & argilosa & floresta & $5 \mathrm{a}$ & $62 \mathrm{a}$ & $61 \mathrm{abc}$ & $24 \mathrm{~b}$ \\
\hline Cambissolo & argilosa & floresta & $4 \mathrm{a}$ & $40 \mathrm{a}$ & $107 \mathrm{bc}$ & $48 \mathrm{a}$ \\
\hline Latossolo & argilosa & campo & $5 \mathrm{a}$ & $68 \mathrm{a}$ & $114 \mathrm{c}$ & $19 \mathrm{~b}$ \\
\hline Cambissolo & argilosa & campo & $3 \mathrm{a}$ & 93 a & $65 \mathrm{abc}$ & $26 \mathrm{~b}$ \\
\hline Latossolo & média & floresta & $3 \mathrm{a}$ & $106 \mathrm{a}$ & $39 \mathrm{a}$ & $14 \mathrm{~b}$ \\
\hline Latossolo & média & campo & $5 \mathrm{a}$ & $64 \mathrm{a}$ & $90 \mathrm{abc}$ & $22 \mathrm{~b}$ \\
\hline Cambissolo & média & campo & $3 \mathrm{a}$ & 89 a & $50 \mathrm{ab}$ & $13 \mathrm{~b}$ \\
\hline C.V. $(\%)$ & & & 31 & 32,3 & 28,6 & 24,2 \\
\hline \multicolumn{7}{|c|}{ Alburno } \\
\hline Cambissolo & média & floresta & 1 & $21 \mathrm{a}$ & $194 \mathrm{~b}$ & $8 \mathrm{a}$ \\
\hline Latossolo & argilosa & floresta & 1 & $28 \mathrm{a}$ & $94 \mathrm{a}$ & $6 \mathrm{a}$ \\
\hline Cambissolo & argilosa & floresta & 1 & $12 \mathrm{a}$ & $95 \mathrm{a}$ & $7 \mathrm{a}$ \\
\hline Latossolo & argilosa & campo & - & $14 \mathrm{a}$ & $148 \mathrm{a}$ & $5 \mathrm{a}$ \\
\hline Cambissolo & argilosa & campo & 1 & $12 \mathrm{a}$ & $64 \mathrm{a}$ & $11 \mathrm{a}$ \\
\hline Latossolo & média & floresta & 1 & $13 \mathrm{a}$ & $36 \mathrm{a}$ & $8 \mathrm{a}$ \\
\hline Latossolo & média & campo & 1 & $10 \mathrm{a}$ & $112 \mathrm{a}$ & $7 \mathrm{a}$ \\
\hline Cambissolo & média & campo & 1 & $18 \mathrm{a}$ & $95 \mathrm{a}$ & $5 \mathrm{a}$ \\
\hline C.V. $(\%)$ & & & - & 77,9 & 39,9 & 37,9 \\
\hline
\end{tabular}

Tabela 5. Coeficientes de correlação (r) entre as variáveis de crescimento do Pinus taeda e estado nutricional das plantas.

Table 5. Correlation coefficients (r) among Pinus taeda growth variables and plant nutritional status.

\begin{tabular}{lccccc}
\hline $\begin{array}{l}\text { Concentração } \\
\text { de nutriente }\end{array}$ & Total & Serraria & Celulose & DAP & $\begin{array}{c}\text { Volume para } \\
\text { serraria }\end{array}$ \\
\cline { 2 - 5 } & \multicolumn{5}{c}{ Acícula } \\
\hline $\mathrm{Zn}$ & 0,81 & 0,90 & 0,87 & 0,79 & 0,88 \\
$\mathrm{P} / \mathrm{Zn}$ & $-0,84$ & $-0,95$ & $-0,88$ & $-0,88$ & $-0,94$ \\
\hline $\mathrm{Fe}$ & Casca & $-0,63$ & $-0,69$ \\
$\mathrm{Zn}$ & $-0,65$ & $-0,70$ & $-0,69$ & \\
\hline
\end{tabular}




\section{CONCLUSÕES}

- K é o nutriente com maior variação de teor nos compartimentos do Pinus taeda nos diferentes sítios, apresentando o sítio mais produtivo os maiores teores desse elemento nas acículas, na casca e no alburno.

- Os sítios mais produtivos apresentam os menores teores de Ca nas acículas das árvores e mostram uma correlação linear positiva muito forte $(\mathrm{r}=0,80)$ com a porosidade total do solo, evidenciando uma dependência da aeração do solo para a absorção de $\mathrm{Ca}$.

- Os sítios mais produtivos apresentam os maiores teores de Zn nas acículas e uma correlação linear positiva muito forte com as variáveis de crescimento. Esse nutriente na casca também se correlaciona positivamente com a altura das árvores.

- A relação $\mathrm{P} / \mathrm{Zn}$ nas acículas das plantas apresenta correlação linear positiva forte com o crescimento das árvores.

\section{REFERÊNCIAS}

BELlOTE, A. J. F.; SILVA, H. D. Técnicas de amostragem e avaliações nutricionais em plantios de Eucalyptus spp. In: GONÇALVES, J. L. M.; BENEDETTI, V. Nutrição e fertilização florestal. Piracicaba: IPEF, 2000. p. 135-166.

CHAVES, R, de Q.; CORREA, G. F. Micronutrientes no sistema solo-Pinus caribaea Morelet em plantios apresentando amarelecimento das acículas e morte de plantas. Revista Árvore, Viçosa, MG, v. 27, n. 6, p. 769-778, 2003.

DEDECEK, R. A.; FIER, I. S. N.; SPELTZ, R.; LIMA, L. C. S. Influência do sítio no desenvolvimento do Pinus taeda aos 22 anos: 1. características físico-hídricas e químicas do solo. A ser publicado.

DEDECEK, R. A.; MENEGOL, O.; BELLOTE, A. F. J. Influência de sistemas de preparo e da fertilidade do solo no teor de nutrientes de Pinus taeda, em área de reforma compactada. In: FERTBIO 2000: Biodinâmica do solo, 2000, Santa Maria, RS. Anais ... Santa Maria, RS: Dept. de Solos/UFSM, 2000. 1 CD-ROM.

EMBRAPA - Empresa Brasileira de Pesquisa Agropecuária. Serviço Nacional de Levantamento e Conservação de Solos. Manual de métodos de análise de solos. 2. ed. Rio de Janeiro, 1997. 212 p.

EMBRAPA/IAPAR- Empresa Brasileira de Pesquisa Agropecuária / Instituto Agronômico do Paraná. Levantamento de reconhecimento dos solos do estado do Paraná. Londrina, PR: IAPAR, 1984. 412p.

FIELDER, H. J.; NEBE, W.; HOFFMANN, F. Forstliche Pflanzenernährung und Düngung. Stuttgart: Gustav Fischer Verlag, 1973. 481 p.

GONÇALVES, J. L. M. Recomendações de adubação para Eucalyptus, Pinus e espécies típicas da Mata Atlântica. Documentos Florestais, Piracicaba, v. 15, p. 1-23, 1995.

HAAG, H. P. Nutrição mineral de Eucalyptus, Pinus, Araucaria e Gmelina no Brasil. Campinas: Fundação Cargill, 1983. 101 p.

HOYLE, M. C.; MADER, D. L. Relationships of foliar nutrients to growth of red pine in Western Massachusetts. Forest Science, Bethesda, v. 10, n. 3, p. 337-347, 1964.

KABATA-PENDIAS, A.; PENDIAS, H. Trace elements in soils and plants. $2^{\text {nd }}$ ed. Boca Raton: CRC, 1992. $31 \mathrm{p}$.

LASO GARICOITS, L. S. Estado nutricional e fatores do solo limitantes do crescimento de Pinus taeda L. em Telêmaco Borba. 128 f. Dissertação (Mestrado em Engenharia Florestal) - Setor de Ciências Florestais, Universidade Federal do Paraná, Curitiba, 1990.

MALAVOLTA, E. Elementos de nutrição mineral de plantas. São Paulo: Agronômica Ceres, 1980. $251 \mathrm{p}$. 
MARSCHNER, H. Mineral nutrition of higher plants. $2^{\text {nd }}$ ed. London: Academic Press, 1995. 889 p.

MENEGOL, O. Índice de sitio e relação entre altura e teores nutricionais das acículas em povoamentos de Pinus elliottii no Segundo Planalto Paranaense. 74 f. Dissertação (Mestrado em Engenharia Florestal) ) - Setor de Ciências Florestais, Universidade Federal do Paraná, Curitiba, 1991.

REISSMANN, C. B. Exigências nutricionais nos plantios de Pinus. Revista da Madeira, Ed. Especial: Pinus, uma alternativa de mercado. Curitiba, v. 12, n. 68, dez., 2002.

REISSMANN, C. B.; WISNEWSKI, C. Aspectos nutricionais de plantios de Pinus. In: GONÇALVES, J. L. M.; BENEDETTI, V. Nutrição e fertilização florestal. Piracicaba: IPEF, 2000. p. 135-166.

RIGATTO, P. A.; DEDECEK, R. A.; MATOS, J. L. M. Influência dos atributos do solo sobre a produtividade de Pinus taeda. Revista Árvore, Viçosa, MG, v. 29, n. 5, p.701-709, 2005.

SANTANA, D. L. Q.; BELlOTE, A. F. J.; DEDECEK, R. A. Ctenarytaina spatulata, Taylor: Água no solo, nutrientes minerais e suas interações com a seca dos ponteiros de eucalipto. Boletim de Pesquisa Florestal, Colombo, n. 46, p. 57-67, 2003.

SARRUGE, J. R.; HAAG, H. P. Análises químicas em plantas.Piracicaba: ESALQ/USP, 1974. 56 p.

SCHOENHOLTZ, S. H.; VAN MIEGROET, H.; BURGER, J. A. A review of chemical and physical properties as indicators of forest quality: challenges and opportunities. Forest Ecology and Management, Amsterdam, v. 138, n. 1-3, p. 335-356, 2000.

SILVEIRA, R. L. V. A.; HIGASHI, E. N.; GONÇALVES, A. N.; MOREIRA, A. Avaliação do estado nutricional do Eucalyptus: diagnose visual foliar e suas interpretações. In: GONÇALVES, J. L. M.; BENNEDETTI, V. (Eds). Nutrição e fertilização florestal. Piracicaba: IPEF, 2000. p. 79-104.

TAMM, C. O. Nutrient Requirements of Forest Stands. International Review Research. v. 1, p. 115$170,1964$.

VOGEL, H. L. M. Crescimento de Pinus taeda L. relacionado a doses de N, P e K, e sua diagnose nutricional pelo DRIS. Revista Ciência Florestal, Santa Maria, v. 13, n. 1, p.182, 2003.

WEAVER, H. A.; JAMISON, V. C. Effects of moisture on tractor tire compaction of soil. Soil Science, Baltimore, v. 71. n. 1, p. 15-23, 1951.

ZÖTTL, H. M.; TSCHINKEL, H. Nutrición y fertilización forestal: una guía práctica. Medellin: Universidad Nacional de Colombia, 1971. 116 p. 